\title{
A NEW SUBCLASS OF BI-UNIVALENT FUNCTIONS ASSOCIATED WITH CHEBYSHEV POLYNOMIAL
}

\author{
O. KARTHIYAYINI, V. SIVASANKARI* \\ Department of Science \& Humanities, PES University, Electronic City, Bangalore, India \\ Corresponding author: sivasankariv@pes.edu \\ Received June 12, 2018
}

\begin{abstract}
In this paper we introduce a new subclass $H_{\Sigma}(k, \lambda, t)$ of analytic bi-univalent functions by using the Chebyshev polynomials. We obtain estimates on initial coefficient bounds for functions in this subclass.

2010 Mathematics Subject Classification. 30C45.

Key words and phrases. bi-univalent functions; coefficient estimates; Chebyshev polynomial; subordination.
\end{abstract}

\section{Introduction}

Let $A$ denote the class of functions of the form:

$$
g(z)=z+\sum_{n=2}^{\infty} b_{n} z^{n}
$$

which are analytic in the open unit disc $U=\{z: z \in \mathbb{C}$ and $|z|<1\}$ and satisfy the normalization condition $g(0)=g^{\prime}(0)=1$.

Let $f(z)$ and $g(z)$ be analytic functions in $\mathrm{U}$, we say that $f(z)$ is subordinate to $g(z)$, written as

$$
f(z) \prec g(z)
$$

if there exist a Schwarz functions $w(z)$ in $\mathrm{U}$, such that $f(z)=g(w(z))$ with $w(0)=0$ and $|w(z)|<1 \quad(z \in U)[6]$

In particular, if the function $g(z)$ is univalent in $U$, then the above subordination is equivalent to

$$
f(0)=g(0)
$$

and

$$
f(U) \subseteq g(U)
$$

(c)2018 Asia Pacific Journal of Mathematics 
Let $\mathrm{S}$ be the class of $\mathrm{A}$ consisting of the functions of the form (1.1) which are also univalent in U. According to Koebe one quarter theorem [6], it is well known fact that every function $g \in S$ has an inverse $g^{-1}$, which is defined by

$$
g^{-1}(g(z))=z(z \in U)
$$

and

where

$$
g^{-1}(w)=w-a_{2} w^{2}+\left(2 a_{2}^{2}-a_{3}\right) w^{3}-\left(5 a_{2}^{3}-5 a_{2} a_{3}+a_{4}\right) w^{4}+\ldots
$$

A function $f(z) \in A$ is said to be bi-univalent in $U$ if both $g(z)$ and $g^{-1}(z)$ are univalent in $U$. Let $\Sigma$ denote the class of all bi-univalent functions in $U$ given by the Taylor-Maclaurin series expansion (1.1). Several recent researchers [2],[7],[5],[8],[9],[3] used Chebyshev polynomial expansions for finding the estimates on the coefficient bounds for the subclasses of biunivalent functions.Hence motivated by their work, we also use Chebyshev polynomial expansion to find the coefficient estimate of our new subclass of biunivalent functions. The significance of Chebyshev polynomial in numerical analysis is increased in both theoretical and practical points of view. Out of four kinds of Chebyshev polynomials, many researchers dealing with orthogonal polynomials of Chebyshev.For a brief history of Chebyshev polynomials of first kind $T_{n}(t)$, the second kind $U_{n}(t)$ and their applications one can refer[1],[4]. The Chebyshev polynomials of the first and second kinds are well known and they are defined by

$$
T_{n}(t)=\cos n \theta
$$

and

$$
U_{n}(t)=\frac{\sin (n+1) \theta}{\sin \theta} \quad(-1<t<1)
$$

where $\mathrm{n}$ denotes the polynomial degree and $t=\cos \theta$.

We define for $g(z)$ in the form(1.1), the following:

$$
\begin{gathered}
F_{\lambda}^{0} g(z)=g(z) \\
F_{0}^{k} g(z)=g(z) \\
F_{\lambda}^{1} g(z)=F_{\lambda} g(z)=(1-\lambda) g(z)+\lambda z g^{\prime}(z)=z+\sum_{n=2}^{\infty}(1+(n-1) \lambda) b_{n} z^{n}
\end{gathered}
$$

and

$$
\begin{gathered}
F_{\lambda}^{2} g(z)=F_{\lambda}\left[F_{\lambda} g(z)\right]=z+\sum_{n=2}^{\infty}\left((1+(n-1) \lambda)^{2} b_{n} z^{n}\right. \\
\left(0 \leq \lambda<\frac{1}{1-n}, k \in \mathbb{N}_{0}=0,1,2, \ldots\right)
\end{gathered}
$$


Hence, it can be easily seen that

$$
F_{\lambda}^{k} g(z)=z+\sum_{n=2}^{\infty}(1+(n-1) \lambda)^{k} b_{n} z^{n}
$$

In the present paper, we introduce the subclass $H_{\Sigma}(k, \lambda, t)$ of bi-univalent functions associated with the linear operator $F_{\lambda}^{k} g(z)$. Also estimates on the coefficients of functions in this class are obtained using Chebyshev polynomial expansions.

Definition 1.1. For $t \in(1 / 2,1], 0 \leq \lambda<\frac{1}{1-n}$ a function $g(z) \in \Sigma$ is said to be in the class $H_{\Sigma}(k, \lambda, t)$, if the following condition are satisfied:

$$
\frac{z\left(F_{\lambda}^{k} g(z)\right)^{\prime}}{F_{\lambda}^{k} g(z)} \prec H(z, t):=\frac{1}{1-2 t z+z^{2}} \quad(z \in U)(k=0,1,2, \ldots z \in U)
$$

and

$$
\frac{w\left(F_{\lambda}^{k} h(w)\right)^{\prime}}{F_{\lambda}^{k} h(w)} \prec H(w, t):=\frac{1}{1-2 t w+w^{2}}, \quad(w \in U),(k=0,1,2, \ldots w \in U)
$$

where $h=g^{-1}$.

We note that if $t=\cos \alpha$, where $\alpha \in(-\pi / 3, \pi / 3)$, then

$$
H(z, t)=\frac{1}{1-2 \cos \alpha z+z^{2}}=1+\sum_{n=1}^{\infty} \frac{\sin (n+1) \alpha}{\sin \alpha} z^{n} \quad(z \in U) .
$$

Thus

$$
H(z, t)=1+2 \cos \alpha z+\left(3 \cos ^{2} \alpha-\sin ^{2} \alpha\right) z^{2}+\ldots(z \in U)
$$

From [10], we can write

$$
H(z, t)=1+U_{1}(t) z+U_{2}(t) z^{2}+\ldots \quad(z \in U, t \in(-1,1))
$$

where

$$
U_{n-1}(t)=\frac{\sin (n \arccos t)}{\sqrt{1-t^{2}}} \quad(n \in N)
$$

are the Chebyshev polynomials of the second kind and we have

$$
U_{n}(t)=2 t U_{n-1}(t)-U_{n-2}(t),
$$

and

$$
U_{1}(t)=2 t, \quad U_{2}(t)=4 t^{2}-1, \quad U_{3}(t)=8 t^{3}-4 t, \quad U_{4}(t)=16 t^{4}-12 t^{2}+1, \ldots
$$

The generating function of the first kind of Chebyshev polynomial $T_{n}(t), t \in[-1,1]$, is given by

$$
\sum_{n=0}^{\infty} T_{n}(t) z^{n}=\frac{1-t z}{1-2 t z+z^{2}} \quad(z \in U)
$$


The first kind of Chebyshev polynomial $T_{n}(t)$ and the second kind of Chebyshev polynomial $U_{n}(t)$ are connected by:

$$
\frac{d T_{n}(t)}{d t}=n U_{n-1}(t) ; \quad T_{n}(t)=U_{n}(t)-t U_{n-1}(t) ; \quad 2 T_{n}(t)=U_{n}(t)-U_{n-2}(t) .
$$

In this present paper, we use the Chebyshev polynomials expansions to provide the initial coefficients of bi-univalent functions in $H_{\Sigma}(k, \lambda, t)$.

\section{Coefficient bounds for the class $H_{\Sigma}(k, \lambda, t)$}

Theorem 2.1. For $0 \leq \lambda<\frac{1}{1-n}$ and $t \in(1 / 2,1]$, let the function $g(z) \in \Sigma$ given by (1.1) be in the class $H_{\Sigma}(k, \lambda, t)$. Then

$$
\begin{gathered}
\left|b_{2}\right| \leq \frac{2 t \sqrt{2 t}}{\sqrt{\left(2(1+2 \lambda)^{k}-2(1+\lambda)^{2 k}\right) 4 t^{2}+(1+\lambda)^{2 k}}} \\
\left|b_{3}\right| \leq \frac{4 t^{2}}{(1+\lambda)^{2 k}}+\frac{t}{(1+2 \lambda)^{k}}
\end{gathered}
$$

and for some $\mu \in R$,

$$
\left|b_{3}-\mu b_{2}^{2}\right| \leq\left\{\begin{array}{c}
\frac{2 t}{2(1+2 \lambda)^{k}}, \\
\text { for }|\mu-1| \leq \frac{\left[(1+2 \lambda)^{k}-(1+\lambda)^{2 k}\right] 8 t^{2}+(1+\lambda)^{2 k}}{8 t^{2}(1+2 \lambda)^{k}} \\
\frac{8(1-\mu) t^{3}}{\left[(1+2 \lambda)^{k}-(1+\lambda)^{2 k}\right] 8 t^{2}+(1+\lambda)^{2 k}}, \\
\text { for }|\mu-1| \geq \frac{\left[(1+2 \lambda)^{k}-(1+\lambda)^{2 k}\right] 8 t^{2}+(1+\lambda)^{2 k}}{8 t^{2}(1+2 \lambda)^{k}}
\end{array}\right.
$$

Proof. Let $g(z) \in \Sigma$ given by (1.1) be in the class $H_{\Sigma}(k, \lambda, t)$. From (1.3) and (1.4), we have

$$
\frac{z\left(F_{\lambda}^{k} g(z)\right)^{\prime}}{F_{\lambda}^{k} g(z)}=1+U_{1}(t) p(z)+U_{2}(t) p^{2}(z)+\ldots
$$

and for its inverse map, $h=g^{-1}$,

$$
\frac{w\left(F_{\lambda}^{k} h(w)\right)^{\prime}}{F_{\lambda}^{k} h(w)}=1+U_{1}(t) q(w)+U_{2}(t) q^{2}(w)+\ldots
$$

for some analytic function

$$
p(z)=c_{1} z+c_{2} z^{2}+c_{3} z^{3}+\ldots \quad(z \in U)
$$

and

$$
q(w)=d_{1} w+d_{2} w^{2}+d_{3} w^{3}+\ldots \quad(w \in U),
$$


such that $p(0)=q(0)=0,|p(z)|<1(z \in U)$ and $|q(w)|<1(w \in U)$.It is well- known that if $|p(z)|<1$ and $|q(w)|<1$, then

$$
\left|c_{j}\right| \leq 1 \quad \text { and } \quad\left|d_{j}\right| \leq 1 \quad \text { forall } \quad j \in N .
$$

From (2.4),(2.5),(2.6)and (2.7), we have

$$
\begin{gathered}
\frac{z\left(F_{\lambda}^{k} g(z)\right)^{\prime}}{F_{\lambda}^{k} g(z)}=1+U_{1}(t) c_{1}(z)+\left[U_{1}(t) c_{2}+U_{2}(t) c_{1}^{2}\right] z^{2}+\ldots \\
\frac{w\left(F_{\lambda}^{k} h(w)\right)^{\prime}}{F_{\lambda}^{k} h(w)}=1+U_{1}(t) d_{1}(w)+\left[U_{1}(t) d_{2}+U_{2}(t) d_{1}^{2}\right] w^{2}+\ldots
\end{gathered}
$$

Equating the coefficients in (2.9) and (2.10), we get

$$
\begin{gathered}
(1+\lambda)^{k} b_{2}=U_{1}(t) c_{1} \\
2(1+2 \lambda)^{k} b_{3}-(1+\lambda)^{2 k} b_{2}^{2}=U_{1}(t) c_{2}+U_{2}(t) c_{1}^{2} \\
-(1+\lambda)^{k} b_{2}=U_{1}(t) d_{1} \\
\left(4 b_{2}^{2}-2 b_{3}\right)(1+2 \lambda)^{2 k}-(1+\lambda)^{2 k} b_{2}^{2}=U_{1}(t) d_{2}+U_{2}(t) d_{1}^{2}
\end{gathered}
$$

From (2.11) and (2.13), we obtain

$$
c_{1}=-d_{1}
$$

and

$$
2(1+\lambda)^{2 k} b_{2}^{2}=U_{1}^{2}(t)\left(c_{1}^{2}+d_{1}^{2}\right)
$$

Also, by using (2.12) and (2.14) we obtain

$$
2\left[2(1+2 \lambda)^{k}-(1+\lambda)^{2 k}\right] b_{2}^{2}=U_{1}(t)\left(c_{2}+d_{2}\right)+U_{2}(t)\left(c_{1}^{2}+d_{1}^{2}\right)
$$

By using (2.16)in (2.17), we get

$$
\left(2(1+2 \lambda)^{k}-(1+\lambda)^{2 k}-\frac{U_{2}(t)}{U_{1}^{2}(t)}(1+\lambda)^{2 k}\right) 2 b_{2}^{2}=U_{1}(t)\left(c_{2}+d_{2}\right) .
$$

From (1.6),(2.8) and (2.18), we have the desired in equality (2.1). Next, by subtracting (2.14) from (2.12), we have

$$
2 a_{3}\left[(1-\lambda) g_{3}+\lambda h_{3}\right]-2 a_{2}^{2}\left[(1-\lambda) g_{3}+\lambda h_{3}\right]=U_{1}(t)\left(c_{2}-d_{2}\right)+U_{2}(t)\left(c_{1}^{2}-d_{1}^{2}\right) .
$$

Further, in view of (2.15), we obtain

$$
b_{3}=b_{2}^{2}+\frac{U_{1}(t)\left(c_{2}-d_{2}\right)}{4(1+2 \lambda)^{k}}
$$


Hence using (2.16)and applying (1.6), we get desired inequality (2.2). Now, by using (2.18) and (2.20) for some $\mu \in R$, we get

$$
\begin{gathered}
b_{3}-\mu b_{2}^{2}=(1-\mu)\left[\frac{U_{1}^{3}(t)\left(c_{2}+d_{2}\right)}{2\left(\left[2(1+2 \lambda)^{k}-(1+\lambda)^{2 k}\right] U_{1}^{2}(t)-U_{2}(t)(1+\lambda)^{2 k}\right)}\right]+\frac{U_{1}(t)\left(c_{2}-d_{2}\right)}{4(1+2 \lambda)^{k}} \\
=\frac{U_{1}(t)}{2}\left[\left(h(\mu)+\frac{1}{2(1+2 \lambda)^{k}}\right) c_{2}+\left(h(\mu)-\frac{1}{2(1+2 \lambda)^{k}}\right) d_{2}\right],
\end{gathered}
$$

where

$$
h(\mu)=\frac{U_{1}^{2}(t)(1-\mu)}{\left[2(1+2 \lambda)^{k}-(1+\lambda)^{2 k}\right] U_{1}^{2}(t)-U_{2}(t)(1+\lambda)^{2 k}} .
$$

So,we conclude that

$$
\left|b_{3}-\mu b_{2}^{2}\right| \leq\left\{\begin{array}{cc}
\frac{2 t}{2(1+2 \lambda)^{k}}, & 0 \leq|h(\mu)| \leq \frac{1}{2(1+2 \lambda)^{k}} \\
2|h(\mu)|(t), & |h(\mu)| \geq \frac{1}{2(1+2 \lambda)^{k}}
\end{array}\right.
$$

This completes the proof.

Taking $\mu=1$ in Theorem (2.1), we get the following consequence.

Corollary 2.2. For $0 \leq \lambda \leq \frac{1}{1-n}$ and $t \in(1 / 2,1]$, let the function $f \in \Sigma$ given by (1.1) be in the class $H_{\Sigma}(k, \lambda, t)$. Then

$$
\left|b_{3}-b_{2}^{2}\right| \leq \frac{2 t}{2(1+2 \lambda)^{k}}
$$

Taking $\lambda=1$ and $\mathrm{k}=1$ in Theorem (2.1), we get the following consequence.

Corollary 2.3. For $t \in(1 / 2,1]$, let the function $f \in \Sigma$ given by (1.1) be in the class $H_{\Sigma}(t)$ Then

$$
\begin{aligned}
\left|b_{2}\right| & \leq \frac{t \sqrt{2 t}}{\sqrt{1-2 t^{2}}} \\
\left|b_{3}\right| & \leq 4 t^{2}+\frac{t}{3}
\end{aligned}
$$

and for some $\mu \in R$,

$$
\left|b_{3}-\mu b_{2}^{2}\right| \leq\left\{\begin{aligned}
\frac{t}{3}, & |\mu-1| \leq \frac{1-2 t^{2}}{6 t^{2}} \\
\frac{2(1-\mu) t^{3}}{1-2 t^{2}}, & |\mu-1| \geq \frac{1-2 t^{2}}{6 t^{2}}
\end{aligned}\right.
$$

Taking $\mu=1$ in Corollary(2.3), we get the following consequence 
Corollary 2.4. For $t \in(1 / 2,1]$, let the function $f \in \Sigma$ given by(1.1) be in the class $H_{\Sigma}(t)$ . Then

$$
\left|b_{3}-b_{2}^{2}\right| \leq \frac{t}{3}
$$

\section{REFERENCES}

[1] E.H.Doha, The first and second kind chebyshev coefiicients of the moments of the general order derivative of an infintely differentiable function, Int. J. Comput. Math., 51 (1994), 21-35.

[2] Hatun Ozlem Guney, G.Murugusundaramoorthy and K.Vijaya , Coefficient bounds for subclasses of biunivalent functions associated with the Chebyshev polynomials, J. Complex Anal., 2017 (2017), Article ID 4150210.

[3] H.Orhan,N.Magesh and V.K.Balaji, Second Hankel determinant for certain class of bi-univalent functions defined by chebyshev polynomials, arXiv:1705.03313v2.

[4] J.C.Mason,Chebyshev polynomials approximations for the L-membrane eigenvalue problem, SIAM J. Appl. Math., 15 (1967), 172-186.

[5] N.Magesh and S.Bulut, Chebyshev polynomial coefficient estimates for a class of anlytic bi-univalent functions related to pseudo-starlike functions, Afr.Mat, 29 (2017), 203-209.

[6] P.L.Duren, Univalent Functions, Grundlehren der Mathematischen Wissenschaften, Springer, New York , 259 (1983).

[7] Sahsene Altinkaya and Sibel Yalcin, On the Chebyshev polynomial coefficient problem of some subclasses of bi-univalent functions, Gulf J. Math.,5(3) (2017), 34-40.

[8] S.Bulut,N.Magesh and C.Abirami, A Comprehensive class of analytic and bi-univalent functions by means of Chebyshev polynomials, J. Fract. Calc. Appl., 8(2) (2017), 32-39.

[9] S.Bulut,N.Magesh and V.K.Balaji,Initial bounds for analytic and bi-univalent functions by means of Chebyshev polynomials, J. Classical Anal., 11(1) (2017), 83-89.

[10] T.Whittaker and G.N.Watson, A course of modern analysis, reprint of the fourth (1927) edition, Cambridge Library, Cambridge Univ. Press,(1996). 